\title{
The Effect of Plating Densities on the Retrieval of Methionine Suppressor Mutations after Ultraviolet or Gamma Irradiation of Aspergillus
}

\author{
By B. R. SCOTT, T. ALDERSON AND D. G. PAPWORTH \\ M.R.C. Radiobiology Unit, Harwell, Didcot, Berkshire
}

(Received 3I October 1972)

INTRODUCTION

The methionine mutational system in Aspergillus detects prototrophic revertants arising from forward mutation at any of six different suppressor gene loci (Scott \& Alderson, 197I). It has been generally assumed that every conidium containing a prototrophic nucleus will form a colony irrespective of the number of auxotrophic or prototrophic conidia on the plate.

Earlier work by Grigg (1952), on Neurospora, using reconstruction experiments, demonstrated that when the background plating density exceeded about $\mathrm{I} \times 10^{7}$ conidia/plate the number of prototrophs retrieved declined and that the conidial concentrations necessary to inhibit prototrophic colony formation varied for different auxotrophic strains. Although the numbers of conidia plated for the detection of revertants in Aspergillus are considerably less than those used by Grigg, it was thought necessary to investigate the effect of different plating densities on the retrieval of the revertants in order that an accurate determination of mutation frequencies could be obtained. The type of reconstruction experiment used by Grigg, using untreated cells, has been avoided in the present study since it seemed advisable to test for the effect of plating density under actual experimental conditions using u.v.- or $\gamma$-irradiated conidia.

\section{METHODS}

The method of preparing inhibitor-depleted spore suspensions of the methGIbiAl strain of Aspergillus nidulans by rotating the phosphate buffer suspensions containing 'Tween 80 ' for $7 \mathrm{~h}$ in a rotating flat, the composition of the media* and methods of irradiation have been described (Scott, Alderson \& Papworth, I972). These procedures, especially the removal of the germination inhibitor, are essential to achieve consistent levels of survival after radiation treatment in this organism (Scott et al. 1972). Immediately before and after treatment the viability and mutation of the population was assayed by plating on the appropriate minimal media. For the viability estimation the minimal medium plates were supplemented with methionine, biotin, tartrate and sodium deoxycholate. These plates were incubated for 2 days at $37{ }^{\circ} \mathrm{C}$ before the visible colonies were counted, whilst plates of a similar composition but lacking methionine and sodium deoxycholate were used to detect revertants of all the phenotypic classes in the same experiment by scoring the plates after 5 days at $37^{\circ} \mathrm{C}$. The mutants appearing at this time were classified as either A (large green colonies), B (brown, heavily pigmented colonies), or C (small green colonies with a white hyline edge) in accordance with the system described by Lilly (1965). Each of these classes of revertants is thought to be produced by mutation at either of two independent gene loci (Lilly, 1965; Scott et al. 197I).

\footnotetext{
* Difco Purified Agar replaced Oxoid no. 3.
} 


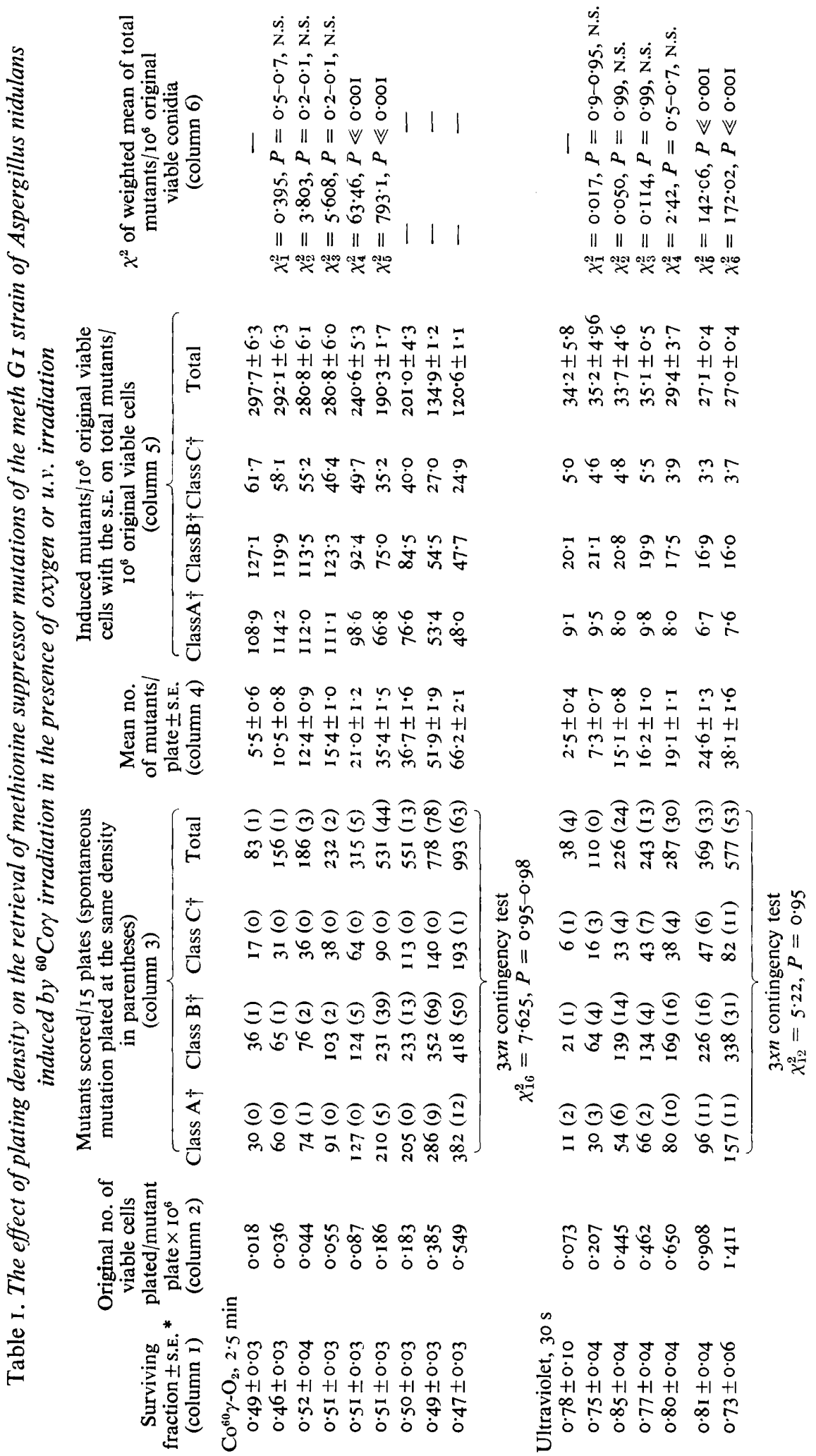



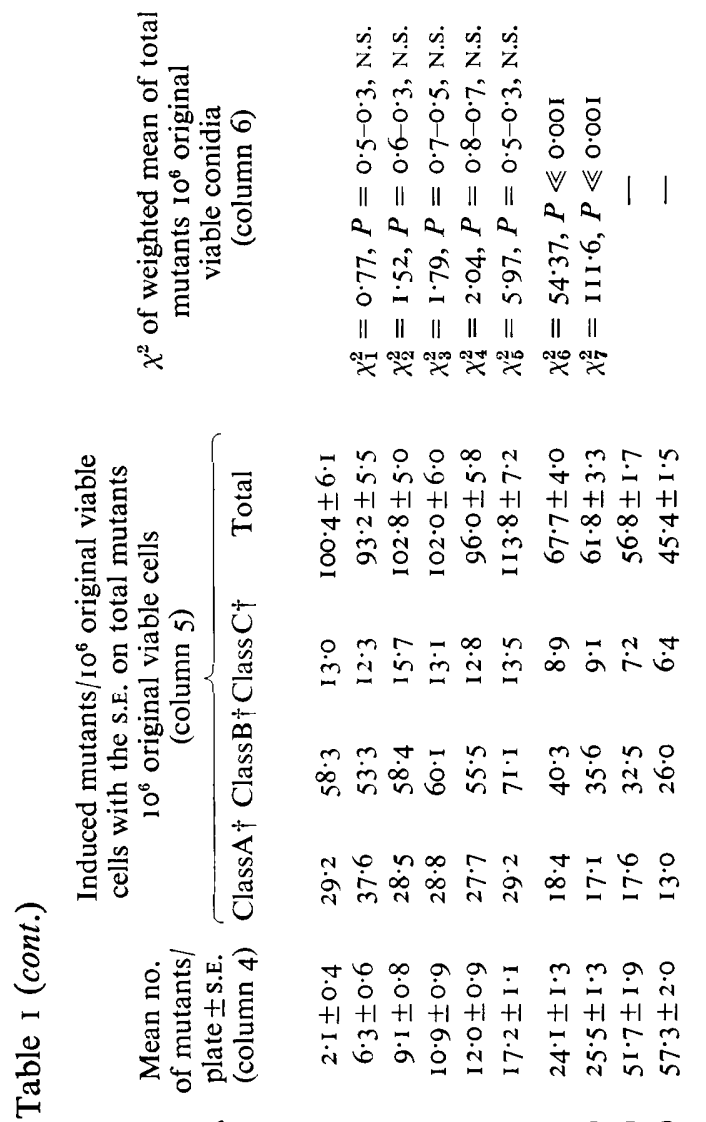

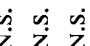

तो तो की

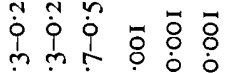

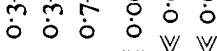

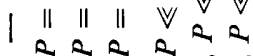

की

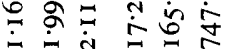

|| || || || || ||

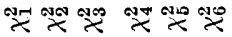

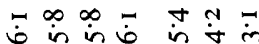

$+1+1+1+1+1+1+1$

- mon $n$

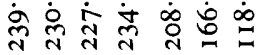

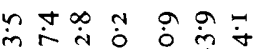

ले ले क्ल क्लि

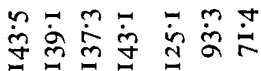

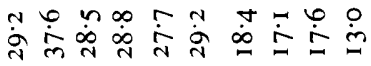

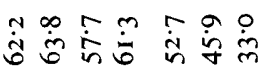

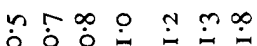

+ீ)

$+1+1+1+1+1+1+1+1+1+1$

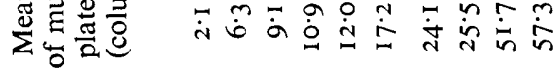

$+1+1+1+1+1+1+1$

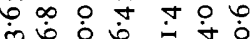

\section{1}

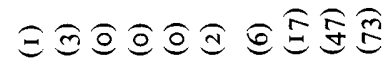

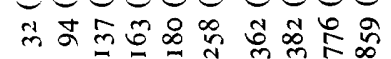

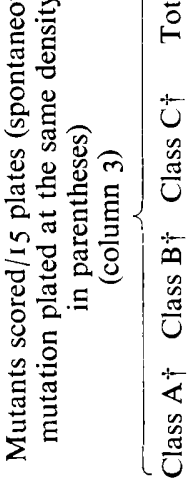

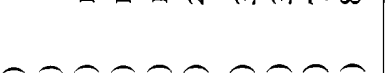

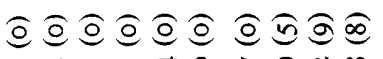

จลำ

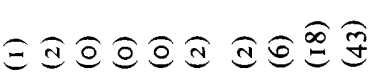

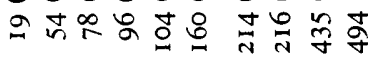

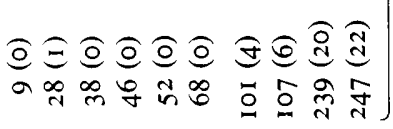

mo

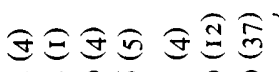

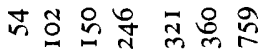

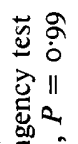

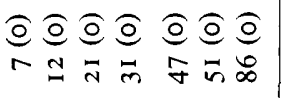

这

\&

氙

응 品

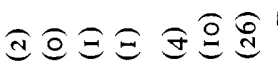

సี

ले

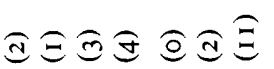

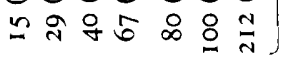

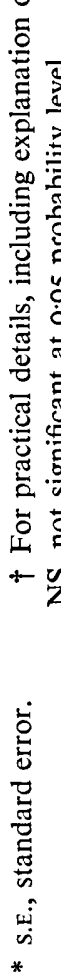

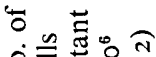

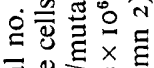

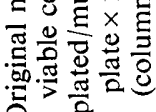

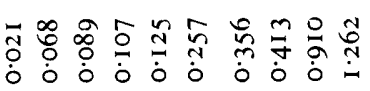

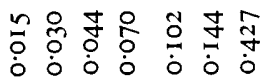
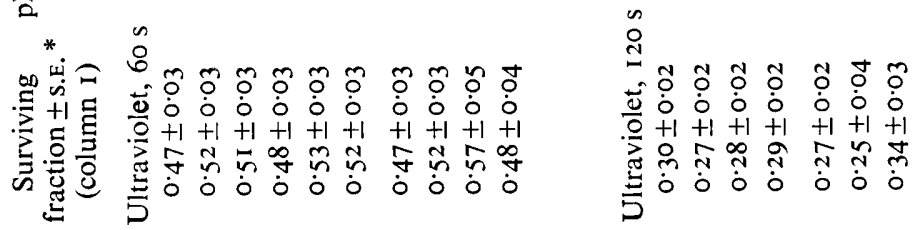


\section{RESULTS}

The results reported in Table I were obtained by irradiating single samples of inhibitordepleted spore suspensions of Aspergillus nidulans with u.v. radiation (dose rate $2 \times 10^{-6}$ $\mathrm{J} / \mathrm{mm}^{2} / \mathrm{s}$ ) for 30,60 and $\mathrm{I} 20 \mathrm{~s}$ in an open Petri dish or $2.5 \mathrm{~min}$ of ${ }^{60} \mathrm{Co} \gamma$-radiation (dose rate $2.58 \mathrm{krad} / \mathrm{min}$ ) whilst oxygen was bubbled through the suspension.

In column I the results are given as the ratio of viable conidia/ml plated immediately after irradiation $\left(N_{l}\right)$ to the originally viable conidia/ml prior to irradiation $\left(N_{0}\right)$. The surviving fractions at a particular dose of radiation were tested for homogeneity by means of $\chi^{2}$ or, if differences between replicate plates were statistically significant, by variance ratio tests. In cases where surviving fractions were satisfactorily homogeneous weighted mean surviving fractions were calculated, each individual being weighted inversely as its estimated variance.

Column 2 lists the number of original viable conidia plated on each of fifteen mutant assay plates to give mutant figures recorded in column 3 . Both the total mutants and the spontaneous mutants scored (recorded in parentheses) were plated at the same originally viable cell density. Replicates for each dose of the various treatments were subjected to a $3 x n$ contingency test, and since there was no statistically significant heterogeneity for the distribution of the three revertant classes for either type of radiation, further statistical analysis was restricted to the total number of induced mutants and not the individual classes of mutants. The exceptional degree of homogeneity obtained for each of the $3 x n$ contingency tests $(P=0.95$ or greater) was expected and is explained later.

Column 4 lists the mean number of mutants/plate together with the corresponding standard error (S.E.), which is based on the assumption that the mutant distribution/plate is Poisson. Induced mutation frequencies (column 5) have been computed by subtracting the number of spontaneous mutations $/ \mathrm{ml}$ from the total number of mutants $/ \mathrm{ml}$ after irradiation, and then dividing by the number of colonies/ml without irradiation $\left(N_{0}\right)$. In computing the S.E. of the total induced mutation frequency the observed number of originally viable colonies/ plate and the mutant distribution/plate have been assumed to follow a Poisson distribution and where necessary the standard error has been increased by a heterogeneity factor, to allow for heterogeneity between replicate plates. Finally, column 6 shows the results of homogeneity tests carried out on the calculated total induced mutation frequencies, by taking them successively two at a time, three at a time, etc. In this way it is possible to determine the mutant density at which the induced mutation frequency begins to decrease.

\section{DISCUSSION}

The results presented for each dose and type of radiation in Table I show that when the number of revertant colonies/plate exceeds 20 , the deduced frequency of mutation induction declines; there is no evidence for a steady decline in mutation frequency as the number of revertants/plate increases. This phenomenon is independent of the type of mutagenic treatment used, the number of originally viable cells plated, the number of surviving auxotrophic conidia/plate and the dose of radiation used. Furthermore, this effect does not alter the specific distribution of the revertant classes for either type or dose of radiation when the number of revertants exceeds the critical level of $20 /$ plate; that is, there is no tendency for the revertant population pressure to affect the retrieval of one or other of the revertant types. Thus the only source of error in the contingency test is that of assigning mutants to a particular class in circumstances where spontaneous mutants are too infrequent to affect the distribution of the induced revertants, or their distribution is in the same ratio for each 
sample, at a particular dose of radiation. Since the three phenotypic classes are easily distinguished from each other, a high probability value is expected for contingency tests in which the limitations on spontaneous mutations apply.

\section{CONCLUSION}

For meaningful quantitative data on radiation-induced mutation to methionine independence in Aspergillus it is, as previously shown, necessary to ensure complete removal of any 'germination inhibitory' substance and, as important, to restrict the number of revertants present/plate to less than 20 . The same situation may well apply to other mutagenic treatments and to spontaneous mutation assayed by this system.

\section{REFERENCES}

GriGg, G. W. (1952). Back mutation assay in micro-organisms. Nature, London 169, 98-100.

LILLY, L. J. (1965). An investigation of the suitability of suppressors of meth I in Aspergillus nidulans for the study of induced and spontaneous mutations. Mutation Research 2, 192-195.

SCOTT, B. R. \& Alderson, T. (197I). The random (non-specific) forward mutational response of gene loci in Aspergillus condia after photosensitisation of near ultraviolet light $(356 \mathrm{~nm})$ by 8 -methoxypsoralen. Mutation Research 12, 29-34.

SCott, B. R., Alderson, T. \& Papworth, D. G. (1972). The effect of radiation on the Aspergillus conidium. I. Radiation sensitivity and a germination inhibitor. Radiation Botany 12, 45-50. 